\title{
Real-World Experience with Coformulated Ledipasvir and Sofosbuvir for HIV-Positive Patients with HCV Genotype 2 Infection: A Multicenter, Retrospective Study
}

Bo-Huang Liou · Hsin-Yun Sun · Chia-Jui Yang · Ling-Shan Syue · Yu-Lin Lee · Hung-Jen Tang •

Hung-Chin Tsai · Chi-Ying Lin · Tun-Chieh Chen · Chun-Yuan Lee · Sung-Hsi Huang •

Chia-Wei Liu · Po-Liang Lu · Shih-Ping Lin · Ning-Chi Wang · Aristine Cheng · Wen-Chien Ko •

Shu-Hsing Cheng (D) C Chien-Ching Hung on behalf of the Taiwan HIV Study Group

Received: January 18, 2021 / Accepted: February 25, 2021 / Published online: March 18, 2021

(C) The Author(s) 2021

\section{ABSTRACT}

Introduction: While coformulated ledipasvir (90 mg)/sofosbuvir (400 mg) (LDV/SOF) is approved for the treatment of hepatitis $C$ virus (HCV) genotype 2 (GT2) infection in Taiwan, Japan, and New Zealand, data regarding its use

Supplementary Information The online version contains supplementary material available at https:// doi.org/10.1007/s40121-021-00424-8.

B.-H. Liou

Department of Internal Medicine, Hsinchu MacKay

Memorial Hospital, Hsinchu, Taiwan

H.-Y. Sun · A. Cheng · C.-C. Hung

Department of Internal Medicine, National Taiwan

University Hospital and National Taiwan University

College of Medicine, Taipei, Taiwan

C.-J. Yang · H.-C. Tsai

School of Medicine, National Yang-Ming University,

Taipei, Taiwan

C.-J. Yang

Department of Internal Medicine, Far Eastern

Memorial Hospital, New Taipei City, Taiwan

L.-S. Syue · W.-C. Ko

Department of Internal Medicine, National Cheng Kung University Hospital and National Cheng Kung University College of Medicine, Tainan, Taiwan

Y.-L. Lee

Department of Internal Medicine, Changhua

Christian Hospital, Changhua, Taiwan for HIV (Human Immunodeficiency Virus)positive patients infected with HCV GT2 are sparse. We aimed to assess the effectiveness and tolerability of LDV/SOF for HIV-positive patients with HCV GT2 coinfection.

Methods: From January 2019 to July 2020, consecutive HIV-positive Taiwanese patients infected with HCV GT2 who received LDV/SOF were retrospectively included for analysis. The effectiveness was determined by sustained virologic response 12 weeks off-therapy (SVR12).

\section{H.-J. Tang}

Department of Internal Medicine, Chi Mei Medical Center, Tainan, Taiwan

\section{H.-J. Tang}

Department of Health and Nutrition, Chia Nan

University of Pharmacy and Sciences, Tainan,

Taiwan

H.-C. Tsai

Department of Internal Medicine, Kaohsiung

Veterans General Hospital, Kaohsiung, Taiwan

C.-Y. Lin

Department of Internal Medicine, National Taiwan University Hospital Yunlin Branch, Yunlin, Taiwan

T.-C. Chen

Department of Internal Medicine, Kaohsiung Municipal Ta-Tung Hospital, Kaohsiung, Taiwan

T.-C. Chen · C.-Y. Lee · P.-L. Lu

Department of Internal Medicine, Kaohsiung Medical University Hospital and Kaohsiung Medical University College of Medicine, Kaohsiung, Taiwan 
Results: Of the 114 patients (mean age, 38.6 years) initiating LDV/SOF during the study period, $0.9 \%$ had liver cirrhosis and $4.4 \%$ were HCV treatment-experienced. All patients had estimated glomerular filtration rate (eGFR) $>30 \mathrm{ml} / \mathrm{min} / 1.73 \mathrm{~m}^{2}$ and were receiving antiretroviral therapy with $98.2 \%$ having CD4 counts $\geq 200$ cells $/ \mathrm{mm}^{3}$ and $93.9 \%$ plasma HIV RNA load $<50$ copies $/ \mathrm{ml}$. Antiretrovirals prescribed included tenofovir alafenamide/emtricitabine in $42.1 \%$, tenofovir disoproxil fumarate (TDF)/emtricitabine $18.4 \%$, other nucleoside reverse transcriptase inhibitors (NRTIs) 39.5\%, non-NRTIs $12.3 \%$, protease inhibitors $13.2 \%$, and integrase inhibitors $74.6 \%$. All patients had undetectable plasma HCV RNA load at the end of treatment, and $96.5 \%$ achieved SVR12 in intention-to-treat analysis. The on-treatment eGFR decline was more pronounced in those receiving TDF-containing antiretroviral therapy (mean change, $-8.33 \mathrm{ml} / \mathrm{min} / 1.73 \mathrm{~m}^{2}$ ), which was reversible after discontinuation of LDV/ SOF. None of the patients interrupted LDV/SOF during the 12-week treatment course.

Conclusion: Similar to the response observed among HIV-negative patients, LDV/SOF is

\section{S.-H. Huang}

Department of Internal Medicine, National Taiwan

University Hospital Hsinchu Branch, Hsinchu,

Taiwan

S.-H. Huang · C.-C. Hung

Department of Tropical Medicine and Parasitology,

National Taiwan University College of Medicine,

Taipei, Taiwan

C.-W. Liu · S.-P. Lin

Department of Internal Medicine, Taichung

Veterans General Hospital, Taichung, Taiwan

N.-C. Wang

Department of Internal Medicine, Tri-Service

General Hospital and National Defense Medical

Center, Taipei, Taiwan

S.-H. Cheng $(\bowtie)$

Department of Infectious Diseases, Taoyuan General Hospital, Ministry of Health and Welfare, Taoyuan,

Taiwan

e-mail: shcheng@mail.tygh.gov.tw

S.-H. Cheng

School of Public Health, Taipei Medical University,

Taipei, Taiwan effective for HIV-positive patients coinfected with HCV GT2.

Keywords: Antiretroviral therapy; Directacting antivirals; End-of-treatment response; Estimated glomerular filtration rate; Ledipasvir; Sofosbuvir; Sustained virologic response

\section{Key Summary Points}

\section{Why carry out this study?}

Despite approval for the treatment of hepatitis $\mathrm{C}$ virus (HCV) genotype 2 (GT2) infection in Taiwan, Japan, and New Zealand, whether coformulated ledipasvir/sofosbuvir (LDV/SOF) will be effective for HIV-positive patients infected with HCV GT2 remains unclear.

\section{What was learned from the study?}

$\mathrm{LDV} / \mathrm{SOF}$ is demonstrated to be effective for HIV-positive patients coinfected with HCV GT2, with a high sustained virologic response rate at 12 weeks off-therapy $(96.5 \%)$, which is similar to that observed among HIV-negative patients.

All included patients completed the 12-week course of LDV/SOF treatment, demonstrating its good tolerability.

The on-treatment decline of estimated glomerular filtration rate was more pronounced in those receiving antiretroviral therapy containing tenofovir disoproxil fumarate (mean change, $-8.33 \mathrm{ml} / \mathrm{min} / 1.73 \mathrm{~m}^{2}$ ), which was reversible after discontinuation of LDV/SOF.

\section{DIGITAL FEATURES}

This article is published with digital features, including a summary slide, to facilitate understanding of the article. To view digital features 
for this article go to https://doi.org/10.6084/ m9.figshare.14096155.

\section{INTRODUCTION}

A global estimate of 71 million people are living with hepatitis $\mathrm{C}$ virus (HCV) and this infection is a major public health threat, particularly in the Asia-Pacific region that bears the largest global burden of death from viral hepatitis [1]. South-East Asia and the Western Pacific region in combination have 24 million people infected by $\mathrm{HCV}$, with disparate prevalence ranging from 0.1 to $4.7 \%$ across countries [1-3]. Approximately 2.3 million (6.2\%) HIV (Human Immunodeficiency Virus)-positive patients have serologic evidence of past or present $\mathrm{HCV}$ infection globally, and the seroprevalence was highest among injection drug users (82.4\%), followed by men who have sex with men (MSM) (6.4\%) and low-risk individuals (2.4\%) [1]. Acquisition of acute $\mathrm{HCV}$ infection through sexual transmission among HIV-positive MSM has been increasingly recognized in Europe, USA, and the Asia-Pacific region [4-7]. Factors facilitating sexual transmission of $\mathrm{HCV}$ among MSM may include unprotective receptive anal sex, chemsex, use of sex toys with mucosal trauma and blood exposure, concurrent sexually transmitted diseases, and the dynamics of social networking resulting from advances in communication technology $[8,9]$.

$\mathrm{HIV} / \mathrm{HCV}$ coinfection is associated with higher risk of fibrosis progression, leading to cirrhosis of the liver and hepatocellular carcinoma if left untreated [10, 11]. HCV disease has become a major cause of death among HIVpositive patients [12]. These people should be diagnosed timely and provided with effective treatment for both HIV and HCV as a priority [13]. Implementation of HCV treatment used to be difficult in the interferon era, however, due to suboptimal treatment responses and safety and tolerability issues. The introduction of direct-acting antivirals (DAAs) has revolutionized the treatment landscape. DAA therapies have resulted in similarly high rates of sustained virologic response (SVR) for HCV infection in patients with and those without HIV infection, making HIV-positive patients no longer a difficult-to-treat population for HCV treatment [14]. Through scaling-up of service coverage of testing, treatment, and prevention, elimination of $\mathrm{HCV}$ is now considered achievable.

Ledipasvir/sofosbuvir (LDV/SOF), the fixeddose combination of NS5A inhibitor and NS5B polymerase inhibitor, is approved for the treatment of patients infected with HCV genotypes $1,4,5$, and $6[15,16]$. The reduced potency of ledipasvir against $\mathrm{HCV}$ genotype 2 (GT2) in vitro has precluded ledipasvir from entering into large-scale clinical trials, and, therefore, LDV/SOF is not routinely used for HCV GT2 infection in Western countries [17]. GT2 accounts for $13 \%$ of HCV infections globally, is commonly seen in some countries in East Asia, Latin American, and Saharan Africa, and has recently been recognized as the leading cause (39.7\%) of acute HCV infection among HIVpositive MSM in Taiwan [6, 7, 18]. Recent studies in New Zealand, Japan, Taiwan, and China have demonstrated high SVR rates (94.8-100\%) and good safety profile in HCV GT2-infected, HIV-negative patients receiving the 8- to 12-week course of LDV/SOF [19-25]. Whether LDV/SOF will be similarly effective for HIV-positive patients with HCV GT2 coinfection remains unclear. We conducted a multicenter, retrospective study to evaluate the effectiveness and tolerability of LDV/SOF in HIV-positive patients coinfected with $\mathrm{HCV}$ GT2.

\section{METHODS}

\section{Study Population and Setting}

In Taiwan, DAAs were conditionally included in the National Health Insurance (NHI) coverage beginning in December 2016 [26]. The National HCV Elimination Program by providing free-ofcharge DAA therapies was subsequently expanded to cover all patients with $\mathrm{HCV}$ viremia, including patients with acute HCV infection, beginning in January 2019. Hepatologists and HIV-treating infectious disease specialists are permitted to screen and treat HIV/HCV-coinfected patients who meet the inclusion criteria. 
The HCV treatment are strictly regulated by the National HCV Elimination Program and the NHI Bureau in Taiwan. Individuals enrolled in the treatment program are required to visit the clinics every 2-4 weeks to refill DAAs during the $\mathrm{HCV}$ treatment course, and at the end of treatment (EOT), and 12 weeks off-therapy. Laboratory examinations are required to be performed at baseline [including serum albumin, total and direct bilirubin, alanine aminotransferase (ALT), aspartate aminotransferase (AST), $\alpha$-fetoprotein, prothrombin time and partial thromboplastin time, hepatitis B virus (HBV) serological markers, HCV genotype, abdominal sonography, and plasma HCV RNA load], week 4 (ALT, AST), the EOT (ALT, AST, plasma HCV RNA load), and 12 weeks off-therapy (total and direct bilirubin, AST, ALT, plasma HCV RNA load). The adherence assessment was conducted by counting the remaining DAA pills at the clinics by treating physicians and case managers. Drug-drug interaction was evaluated prior to LDV/SOF initiation using electronic tools (e.g., website or application software of Liverpool HEP Interactions) [27]. Drugs classified as "should not be coadministered" or "potential clinically significant interaction" with LDV/SOF, such as amiodarone, digoxin, rifampicin, phenytoin, rosuvastatin, and others, were not allowed.

HIV-positive patients in Taiwan are provided with free-of-charge access to combination antiretroviral therapy (cART) and monitoring of plasma HIV RNA load, CD4 lymphocyte count, renal and liver function, lipids, and serological markers of viral hepatitis and viral load, if necessary, according to the national HIV treatment guidelines. HIV care is provided by HIV-treating infectious disease specialists in collaboration with case managers at the designated hospitals around Taiwan.

In this retrospective cohort study, we consecutively included eligible HIV-positive patients aged 20 years or older who were diagnosed with HCV GT2 viremia and initiated a fixed-dose combination tablet of LDV/SOF at the participating hospitals of the Taiwan HIV Study Group between January 2019 and July 2020. Patients were either HCV treatment-naïve or -experienced.

\section{Data Collection}

All data were collected retrospectively from the participating hospitals by reviewing the electronic medical records of the included patients. A standardized data form was used to collect information on demographics (year of birth and gender), clinical characteristics (HIV transmission route and cART regimen before and after initiation of LDV/SOF) and laboratory test results (CD4 count, plasma HIV RNA load, hemogram, and biochemistry). The estimated glomerular filtration rate (eGFR) was assessed with the use of Chronic Kidney Disease Epidemiology Collaboration (CKD-EPI) equation. Blood samples to determine HCV RNA load were obtained at baseline, week 4 and EOT, and 12 weeks off-therapy. All reported adverse events were recorded. This retrospective study was approved by the Institutional Review Board or Research Ethics Committee of each participating hospital [National Taiwan University Hospital (registration number 201003112R), Far Eastern Memorial Hospital (105040-F), Taoyuan General Hospital (TYGH103011), Tri-Service General Hospital (1-105-05-057), Hsinchu Mackay Memorial Hospital (18MMHISO08e), Taichung Veterans General Hospital (CF16114B), Changhua Christian Hospital (160408), National Cheng Kung University Hospital (B-BR-105-038), Chi Mei Medical Center (10505-002), Kaohsiung Veterans General Hospital (VGHKS19-CT4-02), Kaohsiung Medical University Hospital (KMUH-IRB-20110040), and Kaohsiung Municipal Ta-Tung Hospital (KMUHIRB-20130016 and KMUHIRB20130017)], and the requirement for oral or written informed consent was waived.

\section{End Points}

The primary effectiveness end point, analyzed according to the Food and Drug Administration (FDA) snapshot algorithm, was SVR with undetectable HCV RNA 12 weeks off-therapy (SVR12), defined as having plasma HCV RNA load less than the lower limit of quantification (LLOQ, $<30 \mathrm{IU} / \mathrm{ml}) 12$ weeks after completion of LDV/SOF treatment. The primary safety 
endpoint was any adverse event leading to discontinuation of LDV/SOF before completion. The secondary end point was HIV virologic suppression after completing LDV/SOF treatment, which was defined as plasma HIV RNA load $<50$ copies $/ \mathrm{ml}$.

\section{Statistical Analysis}

All analyses were performed using Statistical Program for Social Sciences (SPSS Statistics v.21, IBM, Armonk, NY, USA). The baseline patient characteristics are shown in means with standard deviation (SD) and percentages when appropriate. The SVR12 is shown in number and percentage with 95\% confidence level (CI). The safety profiles and laboratory abnormalities are shown in number and percentages when appropriate. When two proportions were being compared, a chi-square test was used. Fisher's exact test was used when any value in the cells of the contingency table was smaller than 5 . Between-group differences for continuous data were compared using Mann-Whitney $U$ test.

\section{RESULTS}

Between January 2019 and July 2020, a total of 114 HIV-positive patients receiving LDV/SOF for HCV GT2 infection were included. The baseline characteristics are shown in Table 1 . The mean age was 38.6 years (SD 9.1), and $99.1 \%$ of the patients were male. All patients had been receiving cART before initiation of LDV/SOF, with two nucleos(t)ide reverse transcriptase inhibitors (NRTIs) plus a third agent including integrase strand transfer inhibitor (InSTI), protease inhibitor (PI), or non-NRTI. Tenofovir alafenamide (TAF)/emtricitabine and tenofovir disoproxil fumarate (TDF)/emtricitabine were used as the backbone NRTIs in 48 $(42.1 \%)$ and 21 (18.4\%) patients, respectively. Eighty-five (74.6\%) patients used InSTIs as third agent of antiretroviral regimen: 4 patients received coformulated bictegravir/emtricitabine/TAF and the other 44 coformulated elvitegravir/cobicistat/emtricitabine/TAF. Ten patients $(8.8 \%)$ had switch of antiretroviral regimen prior to initiation of $\mathrm{LDV} / \mathrm{SOF}$, including 7 because of concerns about renal function when TDF and LDV/SOF were concurrently used, and 3 because of simplification of antiretroviral regimens. Most of the patients were virally suppressed with HIV RNA load $<50$ copies $/ \mathrm{ml}$ and CD4 lymphocyte count $\geq 200$ cells $/ \mathrm{mm}^{3} \quad(93.9 \%$ and $98.2 \%$, respectively).

The HCV treatment response is shown in Fig. 1. All had HCV RNA < LLOQ at the EOT, and $96.5 \%(110 / 114)$ patients achieved SVR12. Two patients missed the HCV virologic assessment for SVR12 and were counted as virologic failure; the third patient was considered to have a HCV relapse because he reported having neither unprotected sex nor injecting drug use from DAA initiation to SVR12 assessment; and the fourth patient was non-adherent during the 12 -week course of therapy. After excluding the two patients who missed SVR12 assessment, the per-protocol SVR12 rate was 98.2\% (110/112). The trends of AST and ALT levels are shown in Fig. 2. The median AST and ALT level at baseline was 35.0 (IQR 26.0-35.0) IU/L and 51.0 (31.8-92.3) IU/L, respectively, which declined to 21.0 (17.0-24.0) IU/L and 17.0 (12.3-24.8) IU/L, respectively, at SVR12 assessment.

A total of 105 patients had plasma HIV RNA load determined after completion of LDV/SOF treatment and $96(91.4 \%)$ of them remained virally suppressed $(<50$ copies $/ \mathrm{ml}), 2(1.9 \%)$ had plasma HIV RNA load of 50-200 copies $/ \mathrm{ml}$, and $7(6.7 \%)$ had plasma HIV RNA load $>200$ copies/ml. HIV virologic response was not associated with $\mathrm{HCV}$ virologic failure to $\mathrm{LDV} /$ SOF treatment. Compared with the patients who had no switch of antiretroviral regimens, 10 patients who had switch of antiretroviral regimens prior to initiation of $\mathrm{LDV} / \mathrm{SOF}$ treatment had a lower rate of HIV viral suppression at assessment 12 weeks off-therapy (92.6\% [88/ 95] vs. $80.0 \%$ [8/10], $P=0.205)$. Plasma HBV DNA load was available in only 6 patients before initiation of $\mathrm{LDV} / \mathrm{SOF}$, while 2 had follow-up HBV DNA testing after completion of LDV/SOF and both of them had undetectable HBV DNA load $(<20 \mathrm{IU} / \mathrm{ml})$.

No patients reported significant adverse effects leading to premature discontinuation of LDV/SOF. All patients receiving TDF and TAF 
Table 1 Demographic and clinical characteristics of the 114 included HIV-positive patients coinfected with HCV genotype 2 at baseline

\section{Variable}

Age, mean (SD), years

$38.6(9.1)$

Male gender, $n(\%)$

$113(99.1)$

Transmission route of $\mathrm{HCV}$ infection, $n$ (\%)

Sexual transmission

$65(57.0)$

Injecting drug use

Unknown

19 (16.7)

$30(26.3)$

HCV seroconversion within 1 year, $n$ (\%)

$24(21.1)$

Plasma HCV RNA load, mean (SD), $\log _{10}$

$6.3(1.0)$

$\mathrm{IU} / \mathrm{ml}$

HCV treatment-experienced ${ }^{\mathrm{a}}, n(\%)$

DAA

PegIFN \pm ribavirin

Positive HBsAg, $n$ (\%)

Liver cirrhosis, $n$ (\%)

Hepatocellular carcinoma, $n$ (\%)

$0(0)$

eGFR, mean (SD), $\mathrm{ml} / \mathrm{min} / 1.73 \mathrm{~m}^{2}$

$94.4(17.3)$

eGFR $>30 \mathrm{ml} / \mathrm{min} / 1.73 \mathrm{~m}^{2}, n$ (\%)

CD4 lymphocyte count $\geq 200$ cells $/ \mathrm{mm}^{3}$, $n(\%)$

Plasma HIV RNA $<50$ copies/ml, $n(\%)$

Use of cART, $n(\%)$

cART switch before initiation of LDV/SOF treatment, $n$ (\%)

To avoid drug interaction between TDF and $\mathrm{LDV} / \mathrm{SOF}$

Simplification of antiretroviral regimen

Antiretroviral regimen, $n$ (\%)

$\operatorname{Nucleos}(t)$ ide reverse transcriptase inhibitors

TAF-based

TDF-based

Non-TFV-based

$45(39.5)$

Third agent of antiretroviral regimens

$112(98.2)$

$107(93.9)$

$114(100)$

$10(8.8)$

$3(2.6)$
Table 1 continued

\begin{tabular}{lc}
\hline Variable & \\
\hline $\begin{array}{l}\text { Non-nucleoside reverse-transcriptase } \\
\text { inhibitor }\end{array}$ & $14(12.3)$ \\
Protease inhibitor & $15(13.2)$ \\
Integrase strand transfer inhibitor & $85(74.6)$ \\
Dolutegravir & $37(32.5)$ \\
Elvitegravir & $44(38.6)$ \\
Bictegravir & $4(3.5)$ \\
\hline
\end{tabular}

cART combination antiretroviral therapy, $D A A$ direct acting antiviral, $e G F R$ estimated glomerular filtration rate, $H C V$ hepatitis $\mathrm{C}$ virus, $L D V / S O F$ ledipasvir/sofosbuvir, PegIFN pegylated interferon, $S D$ standard deviation, $T A F$ tenofovir alafenamide, $T F V$ tenofovir, TDF tenofovir disoproxil fumarate

${ }^{a}$ Three patients had had treatment with PegIFN \pm ribavirin, one velpatasvir/SOF (VEL/SOF), and the remaining one PegIFN + ribavirin for HCV genotype $1 \mathrm{~b}$ infection and VEL/SOF for HCV genotype $1 \mathrm{~b}$ reinfection when he had achieved HIV viral suppression ( $<20$ copies/ $\mathrm{ml}$ ) with antiretroviral therapy. All these $5 \mathrm{HCV}$ treatment-experienced patients achieved sustained virologic response with LDV/SOF for HCV GT2 infection during the study period

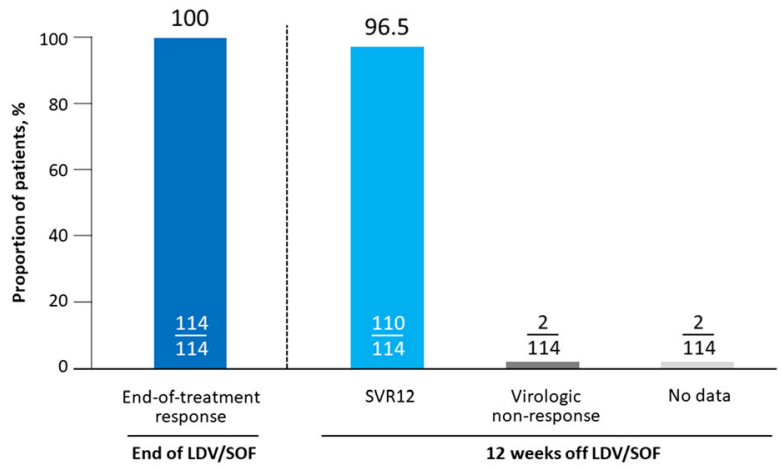

Fig. 1 Treatment response to ledipasvir/sofosbuvir. $L D V /$ SOF ledipasvir/sofosbuvir, SVR12 sustained virologic response 12 weeks off-therapy

had baseline eGFR $\geq 60 \mathrm{ml} / \mathrm{min} / 1.73 \mathrm{~m}^{2}$, with a mean value of 101.8 and $95.7 \mathrm{ml} / \mathrm{min} / 1.73 \mathrm{~m}^{2}$, respectively. Declines in eGFR were observed in 


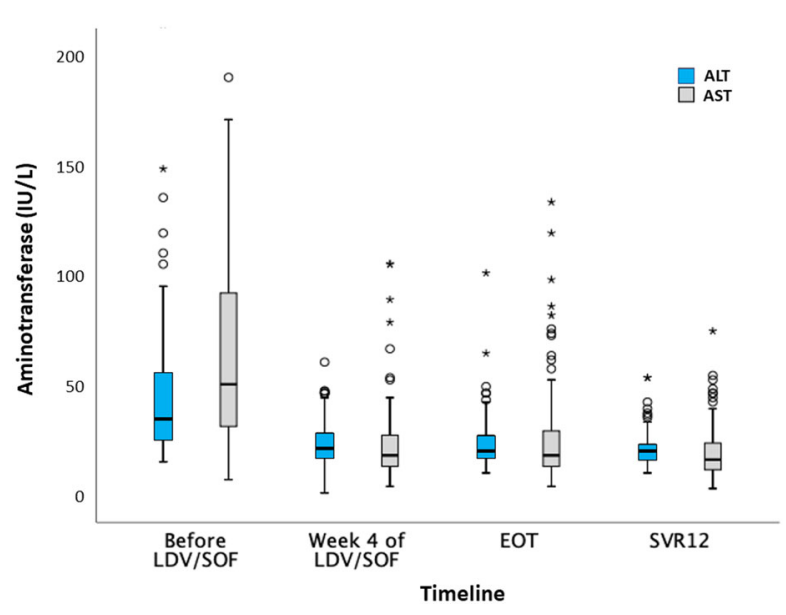

Fig. 2 Sequential change of alanine aminotransferase (ALT) and aspartate aminotransferase (AST). EOT end of treatment, SVR12 sustained virologic response 12 weeks off-therapy

the patients concurrently receiving TDF- or TAF-containing antiretroviral therapy with LDV/SOF (Table 2). Among the patients whose eGFR at baseline and on-treatment were both recorded, the decline of eGFR was more pronounced, but not statistically significant, in those receiving TDF (mean change, $-8.33 \mathrm{ml} /$ $\min / 1.73 \mathrm{~m}^{2}, n=20$ ) than in those receiving TAF $\quad\left(-4.20 \mathrm{ml} / \mathrm{min} / 1.73 \mathrm{~m}^{2}, \quad n=34\right)$ $(P=0.835)$. The changes of eGFR from baseline to the timepoint for assessing SVR12 were small in both groups (TDF, $-0.7 \mathrm{ml} / \mathrm{min} / 1.73 \mathrm{~m}^{2}$, $n=20 ; \quad$ and $\mathrm{TAF}, \quad-3.0 \mathrm{ml} / \mathrm{min} / 1.73 \mathrm{~m}^{2}$, $n=47)$. No patients interrupted LDV/SOF due to declines of eGFR.

\section{DISCUSSION}

Treating patients with HIV/HCV coinfection is a clinical challenge and of great practical importance in our commitment to the elimination of $\mathrm{HCV}$ infection, not only because of increasing incidences of acute HCV infection and reinfection among HIV-positive MSM but also because of complicated issues surrounding drug interactions between DAAs and cART and other concomitant medications or substances in this population. A regimen that is efficacious, safe,

Table 2 Changes of renal function stratified by the use of TAF-, TDF- or non-TFV-containing antiretroviral regimens and different levels of baseline eGFR

\begin{tabular}{lllll}
\hline $\begin{array}{l}\text { eGFR, mean }(\mathbf{S D}), \\
\mathbf{m l} / \mathbf{m i n} / \mathbf{1 . 7 3} \mathbf{~ m}^{\mathbf{2}}\end{array}$ & Before LDV/SOF & During LDV/SOF & SVR12 & Post-SVR12 \\
\hline TDF $(n=21)$ & & & & \\
eGFR $\geq 60(n=21)$ & $101.8(16.1)$ & $94.1(20.7)$ & $100.9(24.0)$ & $99.2(13.7)$ \\
& $(n=21)$ & $(n=20)$ & $(n=20)$ & $(n=7)$ \\
eGFR $<60(n=0)$ & NA & NA & NA & NA \\
TAF $(n=48)$ & & $90.7(15.5)$ & $92.8(16.5)$ & $94.2(15.2)$ \\
eGFR $\geq 60(n=48)$ & $95.7(17.8)$ & $(n=34)$ & $(n=47)$ & $(n=24)$ \\
eGFR $<60(n=0)$ & $(n=48)$ & NA & NA & NA \\
Non-TFV $(n=45)$ & & $91.0(18.8)$ & $87.6(13.9)$ & $90.1(14.4)$ \\
eGFR $\geq 60(n=44)$ & $90.3(15.5)$ & $(n=26)$ & $(n=44)$ & $(n=24)$ \\
eGFR $<60(n=1)$ & $(n=44)$ & 67.4 & 65.0 & NA \\
\hline
\end{tabular}

eGFR estimated glomerular filtration rate, $N A$ not available, $S D$ standard deviation, $T A F$ tenofovir alafenamide, TDF tenofovir disoproxil fumarate, $T F V$ tenofovir 
tolerable, and easy to take is critical for successful treatment scale-up. This retrospective study indicates that a 12-week course of LDV/ SOF treatment was effective in treating HIVpositive patients with HCV GT2 coinfection. We observed a SVR12 rate of $96.5 \%$ in a real-life clinical care setting, while most of the patients remained HIV virally suppressed. All patients completed the 12-week course of LDV/SOF treatment, demonstrating its good tolerability.

The clinical effectiveness of LDV/SOF in patients with HCV GT2 infection is not unexpected. The SVR12 rates following LDV/SOF treatment in HIV-negative patients with $\mathrm{HCV}$ GT2 infection were high (ranging from 94.8 to $100 \%)$ in the published data from China, Taiwan, Japan, and New Zealand (Supplementary Table S1) [19-25]. The SVR12 rate in the intention-to-treat analysis among our $114 \mathrm{HIV} / \mathrm{HCV}$ coinfected patients (96.5\%) is in line with those observed among patients with HCV monoinfection following 12 weeks of LDV/SOF treatment. Our study is the first to address the effectiveness of LDV/SOF for HCV GT2 infection in HIV-positive patients, who were relatively younger than those in previous studies in HCV-monoinfected individuals and in whom only one had compensated cirrhosis of the liver.

The response rate to LDV/SOF in our study is similar to those to pan-genotypic DAAs among patients with HIV/HCV GT2 coinfection $[28,29]$. In the EXPEDITION-2 study conducted exclusively in patients with HIV/HCV infection, treatment with glecaprevir/pibrentasvir (G/P) yielded an SVR12 rate of $100 \%$ for GT2 following $8(n=9)$ or 12 weeks $(n=1)$. In ASTRAL-5 study consisting of $11 \mathrm{HIV} / \mathrm{HCV}$ GT2-coinfected patients without or with compensated cirrhosis, the SVR12 rate was $100 \%$ after completion of treatment with 12 weeks of velpatasvir/SOF (VEL/SOF). While G/P and VEL/SOF have been approved by the United States FDA and the European Medicines Agency, but access to these two regimens in low- and middle-income countries remains limited. With the availability and affordable price (either brand or generic drugs), LDV/SOF could be a reasonable alternative for the treatment of most of the $\mathrm{HCV}$ genotypes other than GT3, which may facilitate the implementation of $\mathrm{HCV}$ elimination programs in resource-limited regions.

Treatment of HIV/HCV-coinfected patients requires continued awareness of and attention to the complex drug-drug interactions that can occur between DAAs and antiretroviral medications [13]. The findings of our study suggest that LDV/SOF is effective and can be used safely in HIV-positive patients coinfected with $\mathrm{HCV}$ GT2 who were receiving a variety of antiretroviral regimens (Table 1). For HIV-positive patients already on cART, HIV-treating physicians tend to choose the DAA regimen in view of patients' antiretroviral regimens. The switch of stable cART regimens in consideration of drug interactions with DAA may put patients at risk of HIV treatment failure, and has been reported to increase the risk of DAA failure [30]. In our study, patients who switched their cART regimen prior to initiation of LDV/SOF treatment had a lower rate of HIV suppression (80\%) than those who continued the same cART regimen $(92.6 \%)$. However, the cause of HIV treatment failure may be multifactorial, and the small case number of the included patients with HIV virologic failure in this study precluded us from making a meaningful inference on the impact of switch of antiretroviral regimens on the virologic response.

The declines of eGFR among our HIV-positive patients receiving LDV/SOF could be multifactorial. Exposure to TDF, rather than TAF, may increase the risk of proximal renal tubulopathy [31]. PIs (such as boosted darunavir, atazanavir, and lopinavir) may cause crystalluria and interstitial nephritis; [32, 33] moreover, PI and cobicistat may also inhibit the secretion of creatinine in the renal tubular cells, leading to declines of eGFR [33]. Controversies exist regarding the potential nephrotoxicity of SOF [34-36]. A prospective study revealed that patients receiving SOF-based DAAs demonstrated trends of on-treatment worsening of eGFR, which were independently related to increasing age, SOF-based DAAs, and more advanced CKD stages [37]. LDV, an inhibitor of P-glycoprotein and BCRP efflux transporters, may increase tenofovir concentration when given with TDF, which may increase the risk of TDF-related nephrotoxicity. In contrast, two 
studies showed that TDF was not associated with increased risk of nephrotoxicity in patients treated with $\mathrm{LDV} / \mathrm{SOF}[38,39]$. In our study, $6.1 \%(n=7)$ patients had a switch of their cART regimens to avoid TDF, while $18.4 \%(n=21)$ continued TDF. We found that the decline in eGFR was greater with TDF- than with TAFcontaining regimens during LDV/SOF treatment $\left(8.33 \mathrm{ml} / \mathrm{min} / 1.73 \mathrm{~m}^{2}\right.$ vs. $6.35 \mathrm{ml} / \mathrm{min} /$ $1.73 \mathrm{~m}^{2}$ ). However, the eGFR declines were reversible in both the TDF and TAF groups at SVR12 assessment, suggesting the removal of the adverse impact of LDV/SOF on renal function and probably the beneficial impact of $\mathrm{HCV}$ eradication on renal function [37]. Compared to baseline, patients receiving TAF had an eGFR decline by $3.0 \mathrm{ml} / \mathrm{min} / 1.73 \mathrm{~m}^{2}$ at SVR12 assessment. The change was minimal, and could be confounded by the boosting agent (cobicistat) that is contained in coformulated elvitegravir/cobicistat/emtricitabine/TAF.

There are several limitations of this study and interpretation of our data should be cautious. First, this was a retrospective, single-arm study, and selection and information bias and confounding could not be avoided. Information on adverse events during the LDV/SOF treatment course could not be evaluated in detail, although none had discontinuation during the treatment course. Second, the case number of included HIV-positive patients in this study remains relatively small when compared to those of the published studies of other DAAs in HIV/HCV-coinfected patients [38, 40]. Third, the included HIV/HCV GT2-coinfected patients in this study were relatively younger than those in prior studies of HCV-monoinfected patients, most of whom might have recently acquired HCV through a sexual route, only one had compensated cirrhosis of the liver, and one had baseline eGFR $<60 \mathrm{ml} / \mathrm{min} / 1.73 \mathrm{~m}^{2}$. Therefore, our findings may not be generalizable to older populations, those with cirrhosis of the liver, and those with chronic kidney disease stage 3 or greater. Fourth, deep sequencing of the HCV NS5A and NS5B coding regions was not performed to determine the emergence of resistance-associated substitutions, and we were not able to differentiate relapse from reinfection in the two patients with documented virologic failure.

\section{CONCLUSIONS}

In conclusion, LDV/SOF for 12 weeks is highly effective, safe, and well tolerated in treating HIV-positive patients with HCV GT 2 coinfection.

\section{ACKNOWLEDGEMENTS}

Funding. No funding was received for this study or writing of this article. The Rapid Service Fee was funded by the authors and a grant from the "Good-Neighbor Fund" of Hsinchu Mackay Memorial Hospital, Taiwan.

Authorship. All named authors meet the International Committee of Medical Journal Editors (ICMJE) criteria for authorship for this manuscript, take responsibility for the integrity of the work as a whole, and have given final approval for the version to be published.

Disclosures. Chien-Ching Hung has received research support from Janssen, Merck, Bristol-Myers Squibb, and ViiV, speaker honoraria from Abbvie, Bristol-Myers Squibb, Gilead Sciences, and ViiV, and serving on advisory boards for Gilead Sciences, Janssen, ViiV, and Abbvie. Bo-Huang Liou, Hsin-Yun Sun, Chia-Jui Yang, Ling-Shan Syue, Yu-Lin Lee, Hung-Jen Tang, Hung-Chin Tsai, Chi-Ying Lin, TunChieh Chen, Chun-Yuan Lee, Sung-Hsi Huang, Chia-Wei Liu, Po-Liang Lu, Shih-Ping Lin, NingChi Wang, Aristine Cheng, Wen-Chien Ko, and Shu-Hsing Cheng have no competing interest to disclose.

Compliance with Ethics Guidelines. This retrospective study was approved by the Institutional Review Board or Research Ethics Committee of each participating hospital (National Taiwan University Hospital [registration number 201003112R], Far Eastern Memorial Hospital [105040-F], Taoyuan General Hospital 
[TYGH103011], Tri-Service General Hospital [1105-05-057], Hsinchu Mackay Memorial Hospital [18MMHIS008e], Taichung Veterans General Hospital [CF16114B], Changhua Christian Hospital [160408], National Cheng Kung University Hospital [B-BR-105-038], Chi Mei Medical Center [10505-002], Kaohsiung Veterans General Hospital [VGHKS19-CT4-02], Kaohsiung Medical University Hospital [KMUHIRB-20110040], and Kaohsiung Municipal TaTung Hospital [KMUHIRB-20130016 and KMUHIRB-20130017]), and the requirement for oral or written informed consent was waived.

Data Availability. The datasets generated during and/or analyzed during the current study are available from the corresponding author on reasonable request

Open Access. This article is licensed under a Creative Commons Attribution-NonCommercial 4.0 International License, which permits any non-commercial use, sharing, adaptation, distribution and reproduction in any medium or format, as long as you give appropriate credit to the original author(s) and the source, provide a link to the Creative Commons licence, and indicate if changes were made. The images or other third party material in this article are included in the article's Creative Commons licence, unless indicated otherwise in a credit line to the material. If material is not included in the article's Creative Commons licence and your intended use is not permitted by statutory regulation or exceeds the permitted use, you will need to obtain permission directly from the copyright holder. To view a copy of this licence, visit http://creativecommons.org/licenses/by$\mathrm{nc} / 4.0 /$.

\section{REFERENCES}

1. World Health Organization. Global Hepatitis Report, 2017. Available at: https://www.who.int/ hepatitis/publications/global-hepatitis-report2017/ en/. Accessed 12 February 2021.

2. Wong MCS, Huang JLW, George J, et al. The changing epidemiology of liver diseases in the Asia-
Pacific region. Nat Rev Gastroenterol Hepatol. 2019;16(1):57-73.

3. Polaris Observatory HCV Collaborators. Global prevalence and genotype distribution of hepatitis $\mathrm{C}$ virus infection in 2015: a modelling study. Lancet Gastroenterol Hepatol. 2017;2(3):161-76.

4. Danta M, Brown D, Bhagani S, et al. Recent epidemic of acute hepatitis $C$ virus in HIV-positive men who have sex with men linked to high-risk sexual behaviours. AIDS. 2007;21(8):983-91.

5. Luetkemeyer A, Hare CB, Stansell J, et al. Clinical presentation and course of acute hepatitis $\mathrm{C}$ infection in HIV-infected patients. J Acquir Immune Defic Syndr. 2006;41(1):31-6.

6. Sun HY, Uemura $H$, Wong NS, et al. Molecular epidemiology of acute HCV infection in HIV-positive patients from Hong Kong, Taipei. Tokyo Liver Int. 2019;39(6):1044-51.

7. Ho SY, Su LH, Sun HY, et al. Trends of recent hepatitis $\mathrm{C}$ virus infection among HIV-positive men who have sex with men in Taiwan, 2011-2018. EClinicalMedicine. 2020;24:100441.

8. Bradshaw D, Matthews G, Danta M. Sexually transmitted hepatitis $C$ infection: the new epidemic in MSM? Curr Opin Infect Dis. 2013;26(1):66-72.

9. Chan DP, Sun HY, Wong HT, Lee SS, Hung CC. Sexually acquired hepatitis $C$ virus infection: a review. Int J Infect Dis. 2016;49:47-58.

10. Sulkowski MS, Mehta SH, Torbenson MS, et al. Rapid fibrosis progression among HIV/hepatitis C virus-co-infected adults. AIDS. 2007;21(16): 2209-16.

11. Macías J, Berenguer J, Japón MA, et al. Fast fibrosis progression between repeated liver biopsies in patients coinfected with human immunodeficiency virus/hepatitis C virus. Hepatology. 2009;50(4): 1056-63.

12. Weber R, Sabin CA, Friis-Møller N, et al. Liver-related deaths in persons infected with the human immunodeficiency virus: the D:A: D study. Arch Intern Med. 2006;166(15):1632-41.

13. Yu ML, Chen PJ, Dai CY, et al. 2020 Taiwan consensus statement on the management of hepatitis C: Part (II) special populations. J Formos Med Assoc. 2020;119(7):1135-57.

14. Sikavi C, Chen PH, Lee AD, Saab EG, Choi G, Saab S. Hepatitis $C$ and human immunodeficiency virus coinfection in the era of direct-acting antiviral agents: no longer a difficult-to-treat population. Hepatology. 2018;67(3):847-57. 
15. Naggie S, Cooper C, Saag M, et al. Ledipasvir and sofosbuvir for HCV in patients coinfected with HIV1. N Engl J Med. 2015;373(8):705-13.

16. He X, Hopkins L, Everett G, et al. Safety and efficacy of ledipasvir/sofosbuvir on hepatitis $C$ eradication in hepatitis $\mathrm{C}$ virus/human immunodeficiency virus co-infected patients. World J Hepatol. 2017;9(30):1190-6.

17. US Food and Drug Administration. Center for Drug Evaluation and Research. Virology review, sofosbuvir and ledipasvir. Available at: https://www. accessdata.fda.gov/. Accessed 12 February 2021.

18. Gower E, Estes C, Blach S, Razavi-Shearer K, Razavi H. Global epidemiology and genotype distribution of the hepatitis $\mathrm{C}$ virus infection. J Hepatol. 2014;61(1 Suppl):S45-57.

19. Gane EJ, Hyland RH, Yang Y, et al. Efficacy of ledipasvir plus sofosbuvir for 8 or 12 weeks in patients with hepatitis $C$ virus genotype 2 infection. Gastroenterology. 2017;152(6):1366-71.

20. Liu CJ, Chuang WL, Sheen IS, et al. Efficacy of ledipasvir and sofosbuvir treatment of HCV infection in patients coinfected with HBV. Gastroenterology. 2018;154(4):989-97.

21. Asahina $\mathrm{Y}$, Itoh $\mathrm{Y}$, Ueno $\mathrm{Y}$, et al. Ledipasvir-sofosbuvir for treating Japanese patients with chronic hepatitis $C$ virus genotype 2 infection. Liver Int. 2018;38(9):1552-61.

22. Lv DD, Wang ML, Chen EQ, et al. A retrospective study of the efficacy of sofosbuvir plus NS5A inhibitors for patients with hepatitis $\mathrm{C}$ virus genotype-2 chronic infection. Eur J Gastroenterol Hepatol. 2019;31(3):382-8.

23. Liu CH, Su TH, Liu CJ, et al. Sofosbuvir-based direct acting antiviral therapies for patients with hepatitis $\mathrm{C}$ virus genotype 2 infection. J Gastroenterol Hepatol. 2019;34(9):1620-5.

24. Ogawa E, Nomura H, Nakamuta M, et al. Ledipasvir and sofosbuvir for 12 weeks for hepatitis $\mathrm{C}$ virus genotype 2 infection: a propensity score matched analysis. Hepatol Res. 2019;50(2):174-81.

25. Chiu HC, Chiu YC, Yang EH, et al. Effectiveness and safety of ledipasvir/sofosbuvir for genotype 2 chronic hepatitis $\mathrm{C}$ infection: real-world experience from Taiwan. J Formos Med Assoc. 2020;120:983.

26. National Health Insurance Administration, Ministry of Health and Welfare, Taiwan. Payment program of direct-acting antroviral agent (in Chinese). Available at: https://www.nhi.gov.tw/Content_List. aspx?n=A4EFF6CD1C4891CA\&topn= 3FC7D09599D25979. Accessed 12 February 2021.
27. Liverpool HEP Interactions. Available at: https:// www.hep-druginteractions.org/. Accessed 16 February 2021

28. Rockstroh JK, Lacombe K, Viani RM, et al. Efficacy and safety of glecaprevir/pibrentasvir in patients coinfected with hepatitis $C$ virus and human immunodeficiency virus type 1 : the EXPEDITION-2 study. Clin Infect Dis. 2018;67(7):1010-7.

29. Wyles D, Bräu N, Kottilil S, et al. Sofosbuvir and velpatasvir for the treatment of hepatitis $C$ virus in patients coinfected with human immunodeficiency virus type 1: an open-label, phase 3 study. Clin Infect Dis. 2017;65(1):6-12.

30. Falade-Nwulia O, Sutcliffe C, Moon J, et al. High hepatitis $\mathrm{C}$ cure rates among black and nonblack human immunodeficiency virus-infected adults in an urban center. Hepatology. 2017;66(5):1402-12.

31. Gupta SK, Post FA, Arribas JR, et al. Renal safety of tenofovir alafenamide vs. tenofovir disoproxil fumarate: a pooled analysis of 26 clinical trials. AIDS. 2019;33(9):1455-65.

32. Mocroft A, Lundgren JD, Ross M, et al. Cumulative and current exposure to potentially nephrotoxic antiretrovirals and development of chronic kidney disease in HIV-positive individuals with a normal baseline estimated glomerular filtration rate: a prospective international cohort study. Lancet HIV. 2016;3(1):e23-32.

33. Yombi JC, Pozniak A, Boffito M, et al. Antiretrovirals and the kidney in current clinical practice: renal pharmacokinetics, alterations of renal function and renal toxicity. AIDS. 2014;28(5):621-32.

34. Saxena V, Khungar V, Verna EC, et al. Safety and efficacy of current direct-acting antiviral regimens in kidney and liver transplant recipients with hepatitis C: results from the HCV-TARGET study. Hepatology. 2017;66(4):1090-101.

35. Mallet V, Parlati L, Dorval O, et al. Estimated glomerular filtration rate variations and direct acting antivirals treatment for chronic hepatitis C: a retrospective longitudinal study. J Hepatol. 2018;68:S22.

36. Okubo T, Atsukawa M, Tsubota A, et al. Efficacy and safety of ledipasvir/sofosbuvir for genotype $1 \mathrm{~b}$ chronic hepatitis $\mathrm{C}$ patients with moderate renal impairment. Hepatol Int. 2018;12(2):133-42.

37. Liu CH, Lee MH, Lin JW, et al. Evolution of eGFR in chronic HCV patients receiving sofosbuvir-based or sofosbuvir-free direct acting antivirals. J Hepatol. 2019;72:839. 
38. Bhattacharya D, Belperio PS, Shahoumian TA, et al. Effectiveness of all-oral antiviral regimens in 996 human immunodeficiency virus/hepatitis $\mathrm{C}$ virus genotype 1-coinfected patients treated in routine practice. Clin Infect Dis. 2017;64(12):1711-20.

39. Soeiro C, Goncalves CAM, Marques MSC, et al. Glomerular filtration rate change during chronic hepatitis $\mathrm{C}$ treatment with Sofosbuvir/Ledipasvir in $\mathrm{HCV} / \mathrm{HIV}$ Coinfected patients treated with Tenofovir and a boosted protease inhibitor: an observational prospective study. BMC Infect Dis. 2018;18(1):364.

40. Bischoff J, Mauss S, Cordes C, et al. Rates of sustained virological response 12 weeks after the scheduled end of direct-acting antiviral (DAA)based hepatitis $\mathrm{C}$ virus (HCV) therapy from the National German HCV registry: does HIV coinfection impair the response to DAA combination therapy? HIV Med. 2018;19(4):299-307. 